\title{
Retinal microglia are critical for subretinal neovascular formation
}

\author{
Ayumi Usui-Ouchi, ${ }^{1,2}$ Yoshihiko Usui, ${ }^{1,3}$ Toshihide Kurihara, ${ }^{1,4}$ Edith Aguilar, ${ }^{1}$ Michael I. Dorrell,,,6 \\ Yoichiro Ideguchi, ${ }^{1}$ Susumu Sakimoto, ${ }^{1,7}$ Stephen Bravo, ${ }^{1,8}$ and Martin Friedlander ${ }^{1,5}$ \\ 'Department of Molecular Medicine, The Scripps Research Institute, La Jolla, California, USA. ${ }^{2}$ Department of \\ Ophthalmology, Juntendo University School of Medicine, Tokyo, Japan. ${ }^{3}$ Department of Ophthalmology, Tokyo Medical \\ University, Tokyo, Japan. ${ }^{4}$ Department of Ophthalmology, Keio University, Tokyo, Japan. ${ }^{5}$ Lowy Medical Research Institute, \\ La Jolla, California, USA. 'Point Loma Nazarene University, San Diego, California, USA. 'Department of Ophthalmology, \\ Osaka University, Osaka, Japan. ${ }^{8}$ Children's Hospital Los Angeles, Los Angeles, California, USA.
}

\begin{abstract}
Abnormal subretinal neovascularization is a characteristic of vision-threatening retinal diseases, including macular telangiectasia (MacTel) and retinal angiomatous proliferation (RAP). Subretinal neovascular tufts and photoreceptor dysfunction are observed in very-low-density lipoprotein receptor $\left(V I d I r^{-}\right)$mutant mice. These changes mirror those observed in patients with MacTel and RAP, but the pathogenesis is largely unknown. In this study, we show that retinal microglia were closely associated with retinal neovascular tufts in $\mathrm{VIdII}^{-1}$ mice and retinal tissue from patients with MacTel; ablation of microglia/macrophages dramatically prevented formation of retinal neovascular tufts and improved neuronal function, as assessed by electroretinography. VIdIr-1- mice with retinal pigmented epithelium-specific (RPE-specific) Vegfa had greatly reduced subretinal infiltration of microglia/macrophages, subsequently reducing neovascular tufts. These findings highlight the contribution of microglia/macrophages to the pathogenesis of neovascularization, provide valuable clues regarding potential causative cellular mechanisms for subretinal neovascularization in patients with MacTel and RAP and suggest that targeting microglia activation may be a therapeutic option in these diseases.
\end{abstract}

Conflict of interest: The authors have declared that no conflict of interest exists.

Copyright: ( 2020 , American Society for Clinical Investigation.

Submitted: February 17, 2020

Accepted: April 30, 2020

Published: June 18, 2020

Reference information: /CI Insight. 2020;5(12):e137317.

https://doi.org/10.1172/jci.

insight.137317.

\section{Introduction}

Pathological angiogenesis in the eye is a characteristic of the vast majority of diseases resulting in irreversible vision loss (1). Retinal angiomatous proliferation (RAP) (a subform of neovascular age-related macular degeneration $[\mathrm{AMD}]$ ) and macular telangiectasia (MacTel) are both characterized by abnormal retinal neovascularization (NV), which leads to loss of central vision $(2,3)$. Unlike most forms of AMD, the vascular changes in patients with MacTel and RAP originate in the intraretinal vasculature, rather than the choroid, and invade the normally avascular layer of photoreceptor cells, eventually developing choroidal NV and retinal-choroidal anastomoses $(4,5)$. The underlying molecular causes of the subretinal NV in these diseases are incompletely understood and may vary between diseases. Currently, the most commonly used therapies are aimed at reducing the activity of VEGF in order to reduce retinal NV. While effective for treating NV AMD and diabetic macular edema, there are issues with anti-VEGF-based therapies, because they are effective only in a subset of patients, elicit off-target effects by blocking VEGF activity $(6,7)$, and can exacerbate photoreceptor dysfunction by inhibiting the neurotrophic effect of VEGF (8-10). In addition, anti-VEGF therapy is ineffective in the treatment of patients with MacTel and, in fact, may be detrimental $(11,12)$. For patients with RAP, anti-VEGF therapies have short-term benefit, but they are not efficacious in the long term (13). Therefore, alternative treatment options are necessary, but these are dependent on better understanding of underlying cellular mechanisms driving the pathological NV.

The very-low-density lipoprotein receptor ( Vldlr) mutant $\left(V l d l r^{--}\right)$mouse represents a murine model of subretinal NV that is commonly used to model the vascular features of MacTel and RAP. Subretinal NV in $V_{l d l r^{-1}}$ mice originates from the intraretinal vasculature, and electroretinography (ERG) analyses reveal photoreceptor impairments, which are particularly striking in cones $(14,15)$. The mechanisms that initiate and direct normal blood vessels to the outer nuclear layer (ONL) are not fully understood. Using immunohistochemistry, we detected microglia/macrophages in areas where subretinal NV tufts subsequently form 
in $V_{l d l r^{-1}}$ mice, just before formation of subretinal NV and colocalized to NV at stages associated with maintenance of subretinal NV. In addition, we detected cells expressing microglial/macrophage markers near NV sites of patients with MacTel. These findings may be significant, because both macrophages and microglia are well recognized to play roles in physiological and pathological retinal angiogenesis (16). Using genetic and pharmacologic approaches, we observed significantly fewer NV tufts in the Vldlr ${ }^{-1}$ mouse and significantly improved ERG recordings when macrophages and microglia were depleted. In contrast, specific VEGF knockout in retinal pigmented epithelium (RPE) resulted in reduced subretinal localization of microglia and prevented subretinal NV tuft formation. These data further demonstrate the key role of microglia activation in driving formation of subretinal NV in the $V l d l r^{--}$mouse model. These insights may provide valuable clues regarding the pathogenesis of diseases with subretinal NV and inform further potential targets for therapeutic intervention in these degenerative eye diseases.

\section{Results}

Retinal microglia are associated with retinal NV tufts in Vldlr ${ }^{--}$mice. To investigate the relationship between sub-

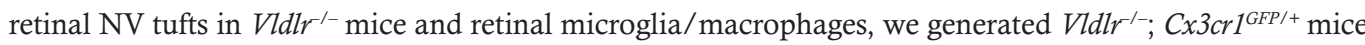
(17). $C \times 3 \mathrm{cr}^{\mathrm{GFP} /+}$-knockin transgenic mice show GFP expression in myeloid cells, including monocytes, dendritic cells, macrophages, and microglia (17). In healthy retina, microglia are known to localize near the vasculature in the inner plexiform layer (IPL), outer plexiform layer (OPL), and ganglion cell layers (GCLs) (18). Neither GFP-positive cells nor GS-lectin-positive vessels were observed in the ONL and subretinal space of $\mathrm{C} \times 3 \mathrm{cr} 1^{G F P /+}$ and $\mathrm{Vldlr}^{/+}$; $\mathrm{C} \times 3 \mathrm{cr}^{\text {GFP/+}}$ mice (Figure 1A and data not shown). However, GFP-positive microglia/macrophages were detected in P27 $\mathrm{Vldlr}^{-1}$; $\mathrm{C} \times 3 \mathrm{cr} 1^{\mathrm{GFP} /+}$ mice, especially in close proximity to the invasive NV vessels and subretinal NV tufts in the outer retinas (Figure 1A). Microglia in ONL and subretinal spaces were round shaped and had short processes, which is characteristic of activated microglia compared with microglia on retinal surfaces (Figure 1A). We also confirmed pathological neovessels and GFP-positive microglia in vivo using deep focus confocal scanning laser ophthalmoscopy images. It was found that GFP-positive cells aggregated around the area of the pathological neovessels visualized by indocyanine green angiography (ICG) at P40 in $V_{l d l}{ }^{--}$; $C \times 3 c r 1^{G F P /+}$ mice (Figure 1B). NV can be observed in patients with MacTel; these subretinal vessels originate from the retinal vasculature, forming retinal-choroidal anastomosis, similar to that seen in $\mathrm{Vldl}^{-1-}$ mice (5). In macular specimens from patients with MacTel type 2, microglia positive for IBA1 (a microglia/macrophage-specific calcium-binding protein) were also found around the abnormal blood vessels within the subretinal space (Figure 1C), suggesting that microglia association with subretinal NV occurs in human disease as well as in the $\mathrm{Vldl}^{-/}$mouse model.

We next set out to investigate the role of microglia during progression of the phenotype in Vldlr $^{\prime-}$; $\mathrm{C} \times 3 \mathrm{cr} 1^{\mathrm{GFP} /+}$ mice. In the $\mathrm{Vldlr^{-1 }}$ retina, $\mathrm{NV}$ starts developing from the retinal vessels, extending into the normally avascular ONL containing the photoreceptor cell bodies by P12, and reaching the RPE and forming subretinal NV by P16 (19). To confirm the roles of retinal microglia in the initiation of NV, we investigated the localization of retinal microglia in $\mathrm{Vldlr}^{--}$; $\mathrm{C} \times 3 \mathrm{cr}^{\mathrm{GFP} /+}$ mice at earlier time points. At P6, neither vessels nor microglia were observed in the ONL and subretinal space. In P10 $\mathrm{Vldlr}^{-1}$; $\mathrm{Cx} 3 \mathrm{cr} \mathrm{IFF}^{\mathrm{GF}}$ mice, before subretinal NV, GFP-positive microglia are observed migrating from OPL into the ONL; this area is normally devoid of microglia. At P13, GFP-positive microglia had migrated into the subretinal space, just ahead of the newly forming neovessels (Figure 1D). The fact that microglia migrate into the ONL and subretinal space before subretinal NV itself suggests that these cells may be promoting subretinal NV rather than reacting to its presence. GFP-positive microglia/macrophages were also observed in RPE of flat-mounted retinas at P14 (Figure 1E), with prominent numbers of GFP-positive microglia/macrophages recruited to the photoreceptor/RPE boundary coalescing around the invading NV tufts at later stages (Figure 1E). Many of these GFP-positive cells localized around subretinal neovessels expressed activated microglia marker CD68 (Supplemental Figure 1; supplemental material available online with this article; https://doi. org/10.1172/jci.insight.137317DS1).

To investigate the molecular basis for the $\mathrm{NV}$ in $\mathrm{Vldl}^{-1-}$ mice, we compared gene expression analyses of 84 different angiogenesis-related genes in retinas from $\mathrm{Vldl}^{-1}$ mice and control $\mathrm{Vldlr}^{{ }^{++}}$littermate mice at P12, just before formation of subretinal NV. The highest changes in mRNA expression between Vldlr ${ }^{-1}$ and heterozygous controls occurred in chemokine (C-C motif) ligand 2 ( $C c l 2$ ), which was increased 4-fold in the $V l d l r^{-1}$ retinas (Figure $1 \mathrm{~F}$ ). $C c l 2$ is primarily secreted by activated microglia to recruit inflammatory monocytes expressing $\operatorname{Cr} 2(20,21)$. 
A

VIdlr ${ }^{1+}$ Cx3cr1 GFP/+
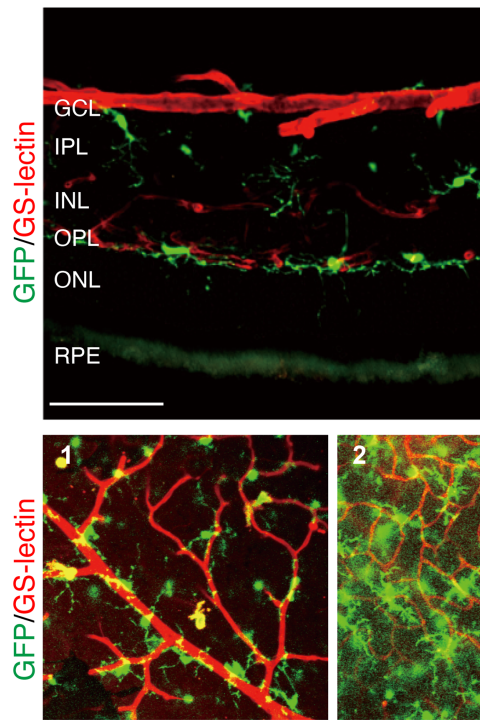

Superficial

B

B
$\overline{\frac{\pi}{0}}$
$\frac{0}{0}$
$\frac{0}{0}$
$\frac{0}{5}$
क

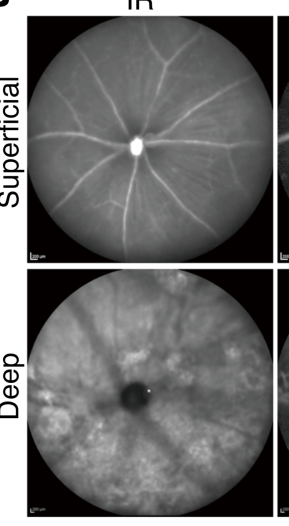

P6

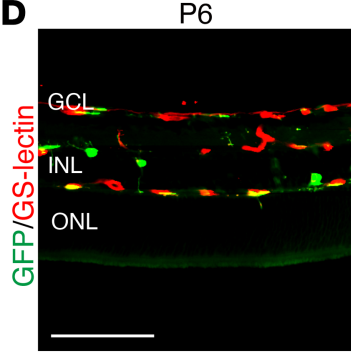

E

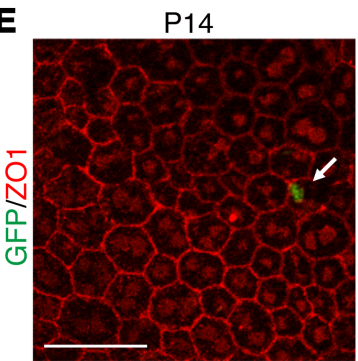

VIdIr $^{--} \mathrm{C} \times 3 \mathrm{Cr} 1^{\mathrm{GFP} /+}$

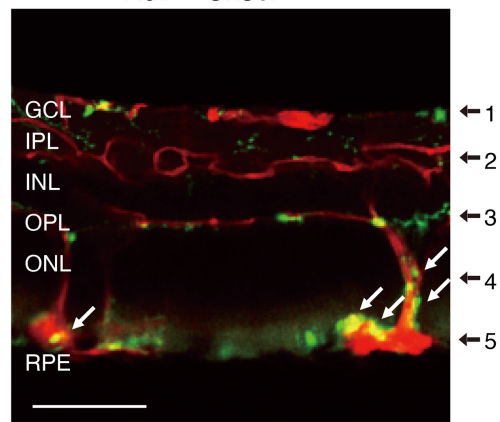

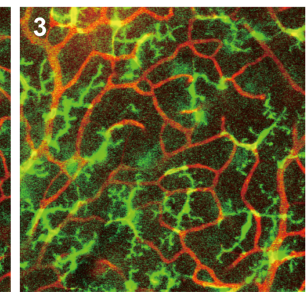

GFP

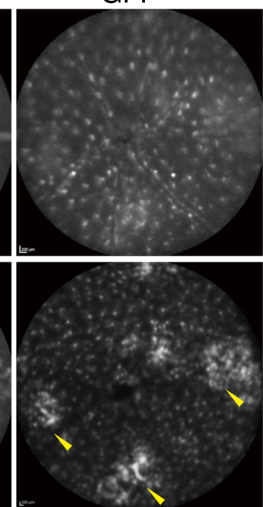

P10

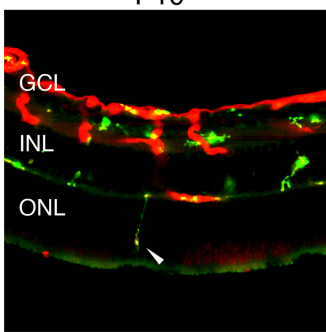

$\mathrm{P} 17$

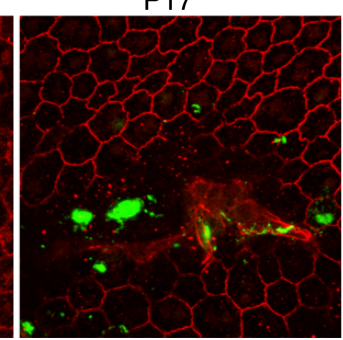

$\mathrm{P} 13$

P27
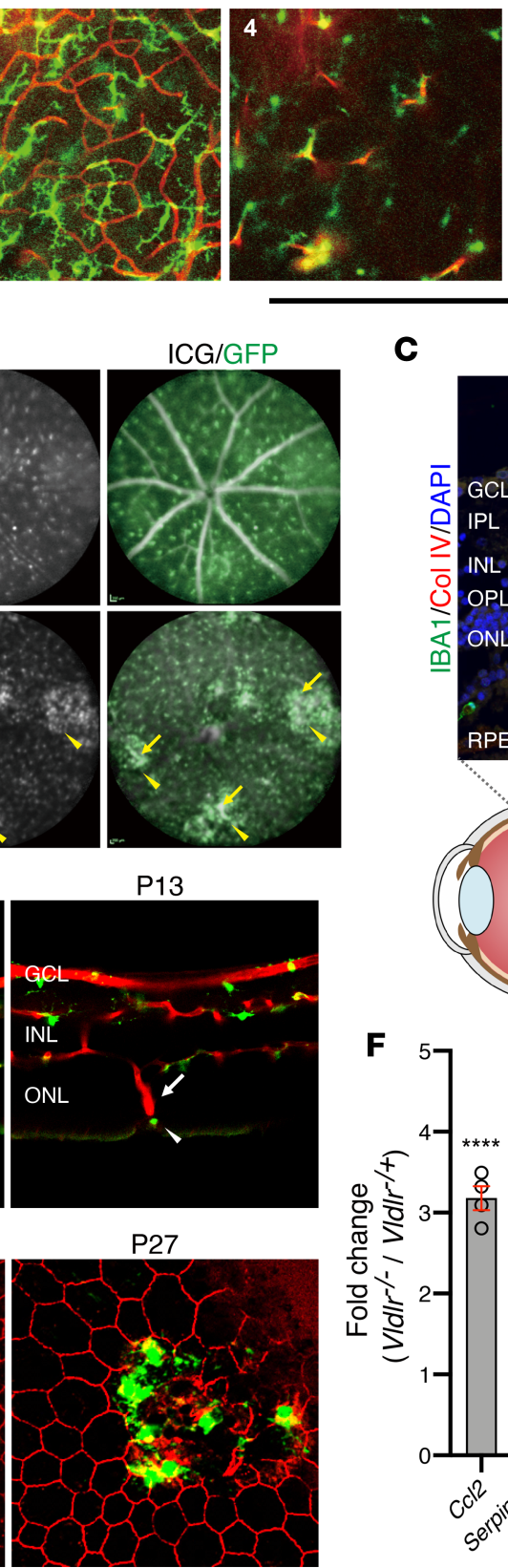

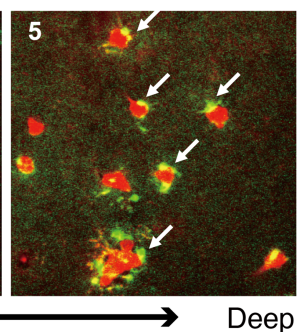

C

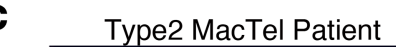

$V_{1 d I r^{-/}} C \times 3 c r 1^{G F P /+}(3 D)$
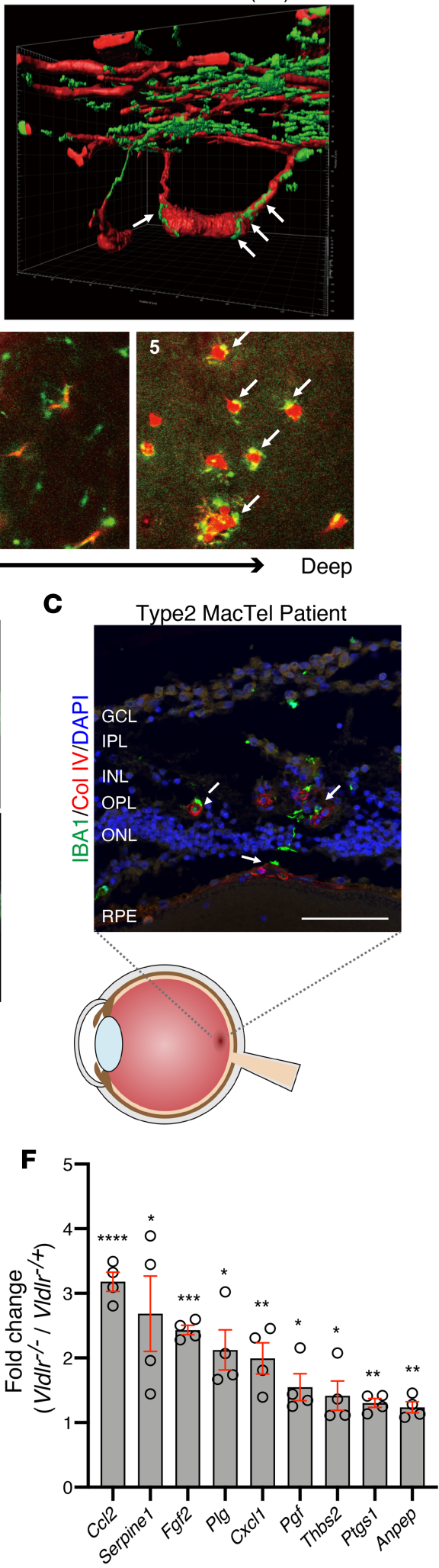
Figure 1. Retinal microglia or macrophages are associated with subretinal neovascular angiomas in VIdIr-/- mice. (A) CS-lectin staining on cryosectioned

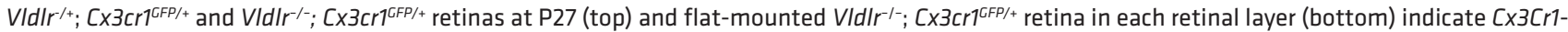
GFP-positive microglia/macrophages (white arrows) in close proximity of abnormal angiomas in the outer nuclear layer and subretinal space in VIdlr-1/;

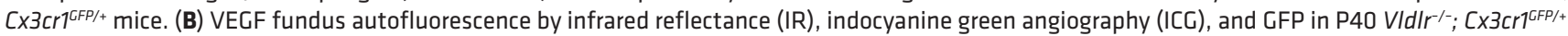
mice showed that the pathological neovascular angiomas (yellow arrows) and the GFP-positive cells were aggregated close to the neovessels (yellow arrowheads). (C) IBA1-positive microglia/macrophages were localized in the outer nuclear layer and subretinal space in proximity of neovessels positive for collagen IV (Col IV; counterstained with DAPI, white arrows) in a retinal section of a macula from a patient with MacTel. (D) CS-lectin staining on cryosectioned $\mathrm{P} 6, \mathrm{P} 10$, or $\mathrm{P} 13 \mathrm{VIdlr}^{--}$; $\mathrm{Cx} 3 \mathrm{Cr} \mathrm{T}^{\mathrm{CFP} /+}$ retinas demonstrates that microglia (arrowheads) migrated into the subretinal space ahead of the neovessels (arrow). (E) Immunostaining for ZO1 in flat-mounted RPE at P14, P17, and P27 of VIdIr-1; Cx3 Cr 1FFP/+ mice. At P14, the first appearance of GFP-positive microglia/macrophages migrated into Z01-positive RPE layer was observed (white arrow) with subsequent NV following. (F) Significantly upregulated genes between VIdIr/- and VIdIr $r^{-1+}$ mice, as analyzed using a PCR array for angiogenesis, are shown $(P<0.05$ and $>1.5$ fold change) The $P$ values were calculated based on a Student's $t$ test of the replicate $2^{(- \text {Delta Ct) }}$ values for each gene in the VIdlr ${ }^{-1}$ group and VIdlr $r^{-1}$ groups. ${ }^{*} P<0.05,{ }^{* *} P<0.01,{ }^{* * *} P<0.001$, ${ }^{* * *} P<0.0001$ ( $n=4$ each). GCL, ganglion cell layer; IPL, inner plexiform layer; INL, inner nuclear layer; OPL, outer plexiform layer; ONL, outer nuclear layer; RPE, retinal pigmented epithelium. Scale bars: $100 \mu \mathrm{m}$ (A, C, and D); $50 \mu \mathrm{m}$ (E).

Taken together, the migration of microglia into the ONL, preceding formation of the subretinal vessels, followed by subsequent colocalization with subretinal NV, suggests that microglia play an important role for the formation and maintenance of these pathological vessels.

Chemically induced microglial ablation reduces retinal $N V$ tufts in Vldlr-1- mice. To further analyze the contribution of microglia to the formation of the pathological NV tufts within the ONL and subretinal space, we used an inhibitor of colony-stimulating factor 1 receptor (CSF-1R). CSF-1R, also known as macrophage colony-stimulating factor 1 receptor (M-CSF-R), is crucial for the viability of microglia, and when CSF-1R is genetically knocked out or pharmacologically inhibited, brain and retina microglia are largely depleted (22-25). Previously, Kubota et al. reported that postnatal systemic injection of CSF-1R inhibitor (Ki20227) from P1 to P4 significantly decreased retinal microglia/macrophages at P4 in mice (24). Daily systemic injections of $\mathrm{Ki} 20227$ (24) from P10 to P16 in $\mathrm{Vldll}^{-1-}$; $\mathrm{C} \times 3 \mathrm{cr} 1^{\mathrm{GFP} /+}$ mice, the time when microglia were found to migrate into the ONL and NV tufts begin subsequent formation, resulted in depletion of GFP-positive retinal microglia/macrophages at P17 (Figure 2, A and B). Strikingly, formation of NV tufts in the Vldlr ${ }^{-1}$ retina was highly suppressed by Ki20227-induced microglia ablation, suggesting that these GFP-positive microglia/macrophage play a critical role in the development of the NV phenotype in $\mathrm{Vldl}^{-1}$ retinas (Figure 2, A-C).

Next, we further tested whether the presence of microglia is critical to the formation of outer retinal/ subretinal NV in $\mathrm{Vldl}^{-1}$ mice by genetic ablation of retinal microglia using cell lineage-specific diphtheria

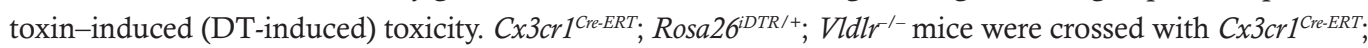
Vldlir-mice and a mouse strain harboring a transgene encoding the human DT receptor (iDTR) within the ROSA26 locus ( $\left.R 26^{\mathrm{DTR}}\right)$. This resulted in mice with $C \times 3 c r 1$-expressing cells (microglia and macrophages) that expressed the iDTR and were therefore susceptible to DT cell death. Administration of DT intraperitoneally from P12 to P14, after subcutaneous injection of 4-hydroxytamoxifen (4-OHT) at P10 and P11 to

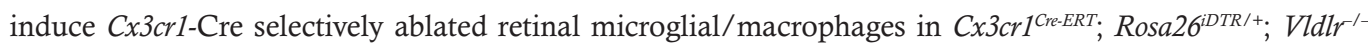
and Rosa26 $6^{\text {DTR/+}}$; Vldl $r^{\prime-}$ mice, and we evaluated NV tufts 9 days later at P23. The numbers of subretinal tufts were significantly reduced in retinas where microglia were genetically ablated compared with controls (Figure 2, D and E). Collectively, chemically or genetically induced retinal microglia/macrophage ablation strongly prevents formation of $\mathrm{NV}$ tufts in Vldl ${ }^{\prime-}$ mice.

Microglia activation increases retinal NV tufts in Vldlr ${ }^{\prime-}$ mice. LPS is a component of the outer membrane of Gram-negative bacteria, and systemic LPS injection leads to increased retinal microglia activation (26). Intraperitoneal LPS injection of $\mathrm{Vldlr}^{-1-} ; \mathrm{C} 3 \mathrm{cr} 1^{\text {GFP/+ }}$ mice at P4, before observed microglia migration and subsequent subretinal NV, resulted in a significant increase in the number of subretinal tufts at P23 as compared with that in $\mathrm{Vldll}^{-1}$; $\mathrm{Cx}_{3 \mathrm{Cr}} \mathrm{CFFP/+}^{\mathrm{GF}}$ mice injected with PBS control (Figure 3, A and C). In LPS-treated Vldlr ${ }^{\prime-}$; $C \times 3 c r 1^{G F P /+}$ retinas, many GFP-positive cells were observed localized to NV tufts compared with those in controls (Figure 3B). However, administration of LPS to $\mathrm{Vldlr}^{-1-}$; $\mathrm{Cx} 3 \mathrm{cr}^{\mathrm{GFP} /+}$ mice at P28, the time when most NV tufts have already formed, did not result in a significant increase in the number of NV tufts 2 weeks later (Figure 3, A and D). These results suggest that the existence and activation of microglia play an important role in the formation of retinal NV in $\mathrm{Vldlr}^{-1-}$ mice.

Microglia/macrophage ablation rescues cone photoreceptor function in Vldlr-- mice. Having found that microglia are critical for initiation of NV tufts, we next tested whether microglia ablation at later stages not only results in reduction of the $\mathrm{NV}$ tuft formation in $V l d l r^{-/}$mice, but also rescues retinal function. Injection 
A
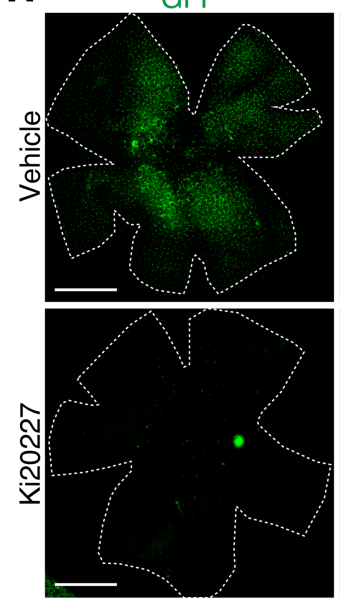

D

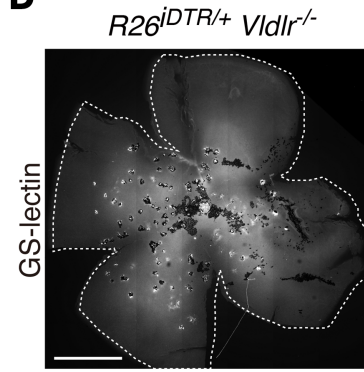

GS-lectin

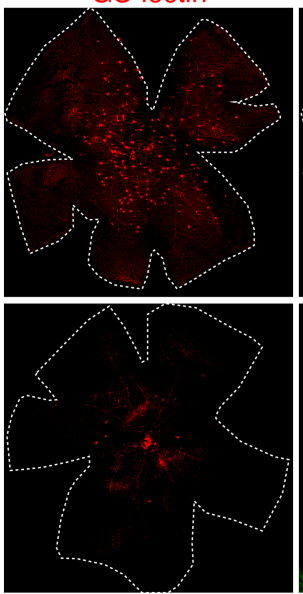

CX3Cr1 Cre-ERT R26 $6^{i D T R /+}$ VIdIIr $^{-/}$

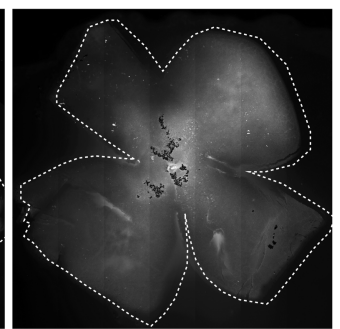

GFP/GS-lectin

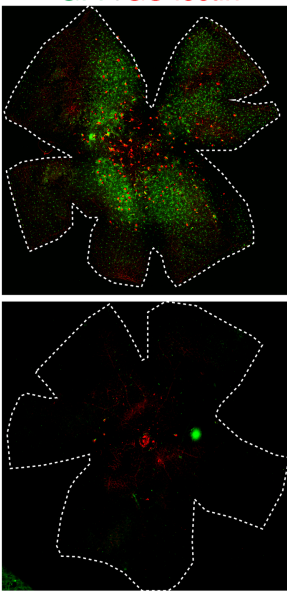

B
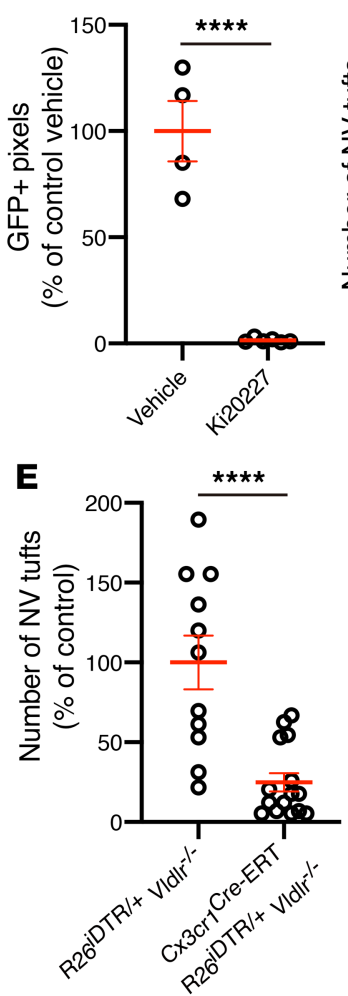

C

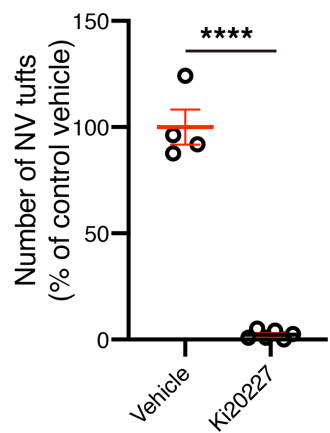

Figure 2. Microglia ablation prevents subretinal neovascularization in VIdIr/- mice. (A-C) Pharmacological microglia ablation using the CSF-1R inhibitor Ki 20227 in VIdlr ${ }^{-1-}$; CX3Cr7 ${ }^{C F P /+}$ mice. (A) Subretinal neovascular (NV) tufts and GFP-positive microglia/macrophages were analyzed using GS-lectin staining in P17 VIdlr ${ }^{-1-}$; CX3 $\mathrm{Cr}^{\mathrm{CFP} /+}$ mice treated with vehicle or Ki20227 from P10 to 16. Both (B) GFP-positive pixels and (C) the number of subretinal NV tufts were significantly reduced in Ki20227-treated mice retina. The $P$ values were calculated using an unpaired 2-tailed $t$ test (vehicle:

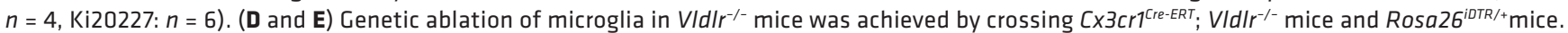

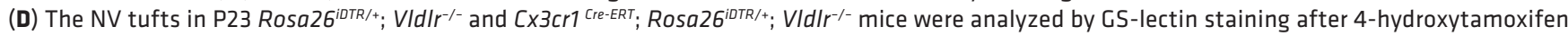
(4HT) treatment at P10 and P11, followed by P12-P14 diphtheria toxin (DT) treatment, demonstrating a marked reduction of NV tufts in Cx3cr1 ${ }^{\mathrm{re}-\mathrm{ERT} \text {; }}$

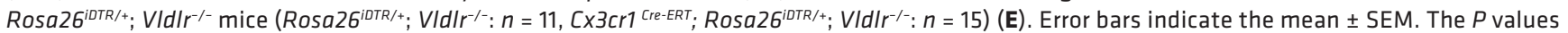
were calculated using an unpaired 2-tailed $t$ test. ${ }^{* * *} P<0.0001$. Scale bars: $1 \mathrm{~mm}$.

of Ki20227 from P10 to P24 resulted in almost complete elimination of NV tufts in Vldlr ${ }^{\prime-}$ mice at P25, similar to the P10-P16 injections previously discussed (Figure 4, A-C, and Figure 2, A and C). Injection of Ki20227 from P17-P24, starting after subretinal NV, resulted in a 80\% reduction of subretinal NV tufts at P25 (Figure 4, A-C). Moreover, when analyzed at P45, NV tufts were still significantly inhibited, despite the fact that the injections were halted 3 weeks earlier (Figure 4, A-C), although total reduction was now only $50 \%$ compared with $80 \%$, suggesting that some NV occurred after injections were stopped. Furthermore, we analyzed retinal function using ERGs to confirm whether reduction of NV tufts by microglia ablation rescues subsequent photoreceptor function defects. Cone photoreceptor dysfunction/death is also characteristic of $\mathrm{Vldlr}^{-1}$ mice and likely occurs secondary to vascular changes (15). ERGs of Vldlr ${ }^{-1}$ mice analyzed at P45 after injection with Ki20227 from P17-P24 and controls analyzed at P45 (Figure 4A) demonstrated rescue of cone photoreceptor function, as assessed by photopic flash and photopic flicker ERGs (Figure 4, D and E). These data demonstrate that retinal function is negatively correlated to subretinal NV tufts and that preventing subretinal NV tufts via microglia ablation can potentially rescue both the pathological vascular phenotype and visual function in $\mathrm{Vldll}^{-1-}$ mice.

VEGF from RPE, but not microglia, regulates microglia activation and tuft formation. We (15), and others $(19,27)$, have reported that Vegfa expression is increased in Vldl $r^{-1}$ retinas at an early stage. We set out to determine whether microglia-derived Vegfa contributed to the NV phenotype. We genetically ablated floxed Vegfa alleles in retinal microglia and macrophages by crossing Cx3cr1 ${ }^{\text {Cre-ERT }}$ or Lys $M^{\text {cre }}$ with $V l d l r^{\prime-}$ mice. LysM is myelomonocytic cell-specific (macrophage, monocyte, and granulocyte) gene; it is known to be expressed in some populations of retinal microglia during development $(28,29)$. 
A

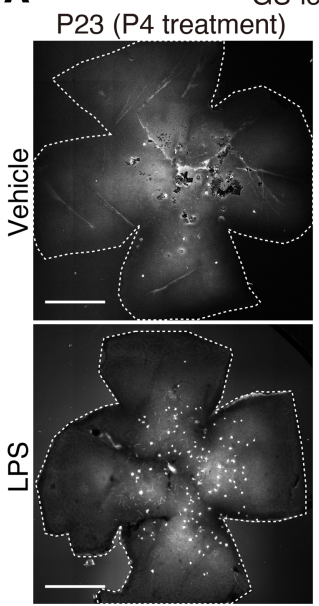

GS-lectin

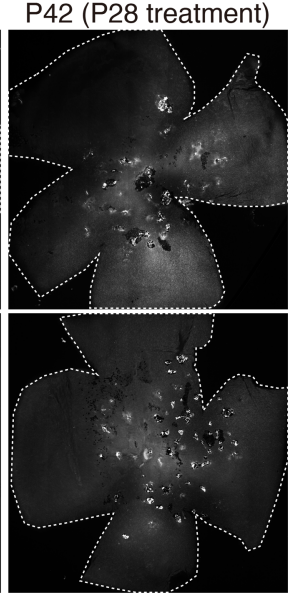

B GFP/GS-lectin

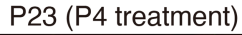

C

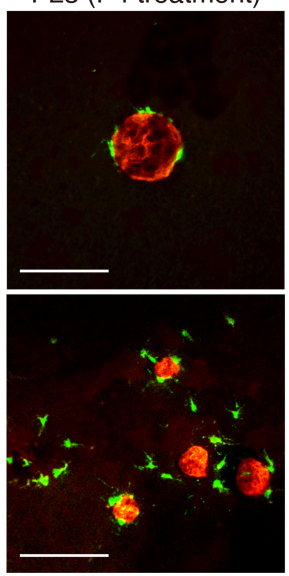

P23 (P4 treatment)

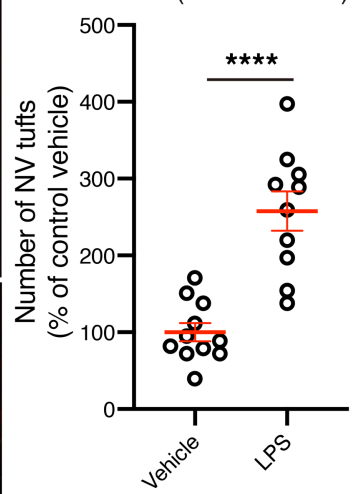

D

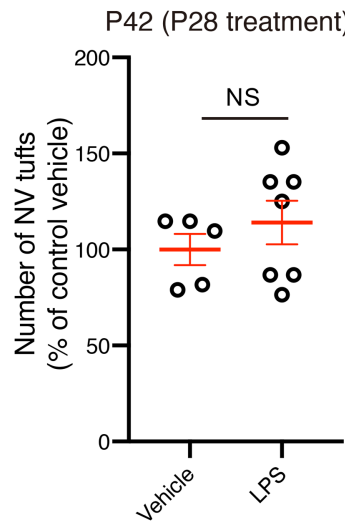

Figure 3. Microglia activation exacerbates subretinal neovascularization in VIdIr/- mice. LPS or vehicle (PBS) was injected subcutaneously into VIdlr ${ }^{-1 /}$; $\mathrm{CX}_{\mathrm{CC}} \mathrm{C}^{\mathrm{CFP} /+}$ mice at $\mathrm{P} 4$ or P28, and then the number of subretinal NV tufts were quantified at P23 or P42, respectively. (A) CS-lectin-stained flat-mounted

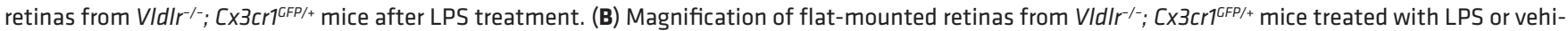
cle at P4. (C and D) LPS treatment at an early stage (P4) significantly increased the number of NV tufts in P23 VIdlr $r^{-1-} ; C^{2} 3 \mathrm{Cr}^{\mathrm{CFP} /+}$ mice, while LPS treatment at a later stage (P28) did not affect the number of subretinal NV tufts compared with vehicle control (C; vehicle: $n=11$, LPS: $n=10, \mathbf{D} ;$ vehicle: $n=5$, LPS: $n$ = 7). Error bars indicate the mean \pm SEM. The $P$ values were calculated using an unpaired 2-tailed $t$ test. ${ }^{* * *} P<0.0001$. Scale bars: $1 \mathrm{~mm}(\mathbf{A}) ; 50 \mu \mathrm{m}(\mathbf{B})$.

On the other hand, $C \times 3 c r 1$ is mainly expressed in microglia and also expressed in perivascular macrophages, which are sparse in the retina $(30,31)$. We observed no significant differences in the number of

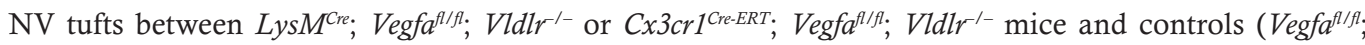
$V l d l r^{--}$) at P23 (Figure 5, A and B), suggesting that microglia-derived Vegfa was not required for the formation of retinal NV tufts in $V l d l r^{-1}$ mice. In fact, ISH analysis of Vegfa and Itgam (CD11b) expression demonstrated that high levels of Vegfa expression were observed in areas of the ONL where NV was prominent but not necessarily correlated with microglia/macrophages (Supplemental Figure 2). This also suggests that other cell types within the subretinal space, besides microglia, are likely to be responsible for substantial VEGF expression. Previous studies have also demonstrated high levels of VEGF expression in the ONL and RPE of $\operatorname{Vldl}^{-1}$ mice $(15,19,32)$.

To determine whether Vegfa expression in RPE is critical for microglia activation and NV formation, we generated RPE-specific Vegfa-deleted Vldlr ${ }^{\prime-}$ mice. In P14 Vldlr ${ }^{-1}$ mice deficient in RPE-derived Vegfa $\left(V M D 2^{C r e} ; V e g f a^{f l f f} ; V l d l r^{-1}\right)$, in which Cre-recombinase was induced at P10, neither NV tufts nor microglia within the ONL were observed, while P14 control mice (Vegfallff; Vldl ${ }^{--}$) showed microglia migration

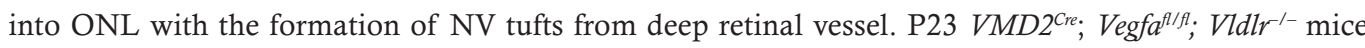
also had reduced formation of subretinal NV tufts as well as reduced migration of microglia/macrophages compared with control Vldlr ${ }^{\prime-}$ mice (Figure 5, C and D), which had substantial subretinal NV tufts with massive microglia/macrophages infiltrates, as expected (Figure 5, C and D). The percentage of IBA1-positive area in the retina was significantly reduced in $V M D 2^{\mathrm{Cre}} ; \mathrm{Vegfa} \mathrm{a}^{f / f l}$; $\mathrm{Vldl}^{-1-}$ mice compared with that in Vegfa $a^{f / f l}$; Vldl $r^{-/}$mice (Supplemental Figure 3). The expression of microglia-related inflammatory genes, such as $C c l 2, I l 1 b, I l 6$, and $T n f a$, was significantly suppressed in retinas from RPE-specific Vegfa-deleted $\mathrm{Vldlr}^{-1-}$ mice compared with that in controls (Figure 5E). The number of subretinal NV tufts at a later stage, P56, after doxycycline treatment at P21 was also significantly reduced in RPE-specific Vegfa deleted Vldlr ${ }^{--}$mice (Figure 5, F and G) While blocking RPE-mediated VEGF expression blocked microglia migration and subsequent subretinal NV, it also resulted in reduced ERG photopic function (Figure 5, H and I). This result was somewhat surprising given our earlier observation that reducing NV rescues the retinal phenotype; it may suggest that the natural upregulation of VEGF in the $\mathrm{RPE}$ of $\mathrm{Vldl}^{\prime-}$ mice is critical for maintenance of the retinal photoreceptors. Taken together, we suggest that Vegfa expression in RPE cells play a key role in microglia/macrophage migration or activation and subsequent subretinal NV formation in $V_{l d l}{ }^{-}$mice. However, Vegfa deletion in RPE can induce impairment of retinal function, further indicating that caution may be warranted when treating retinal diseases with subretinal NV using VEGF inhibition. 


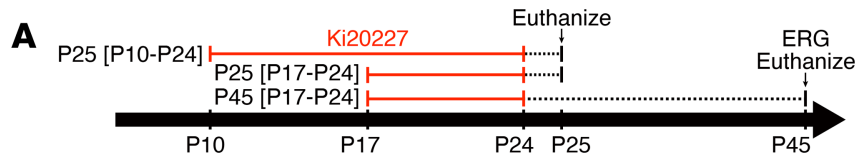

B

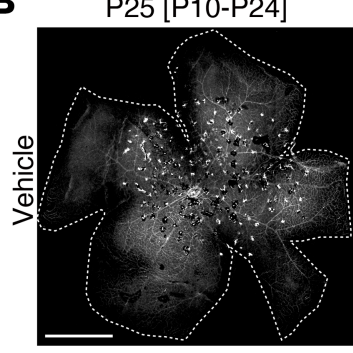

$$
\text { P25 [P17-P24] }
$$
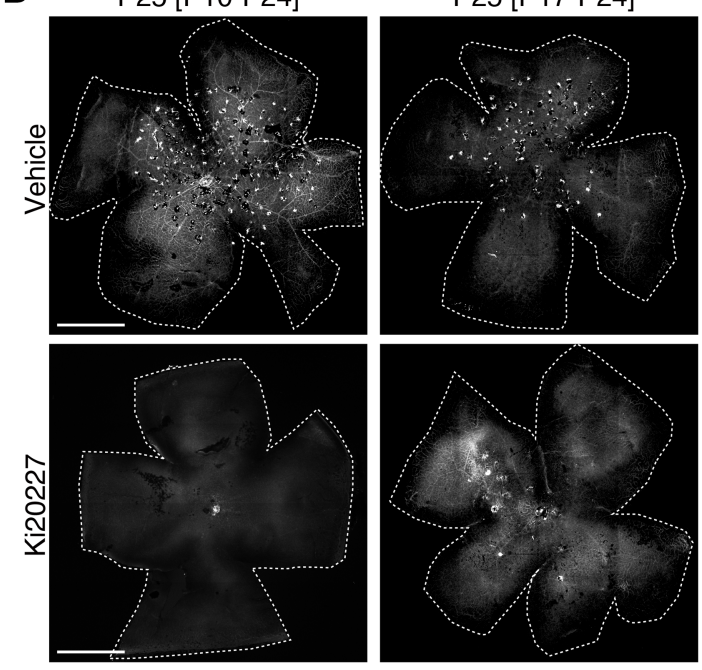

D

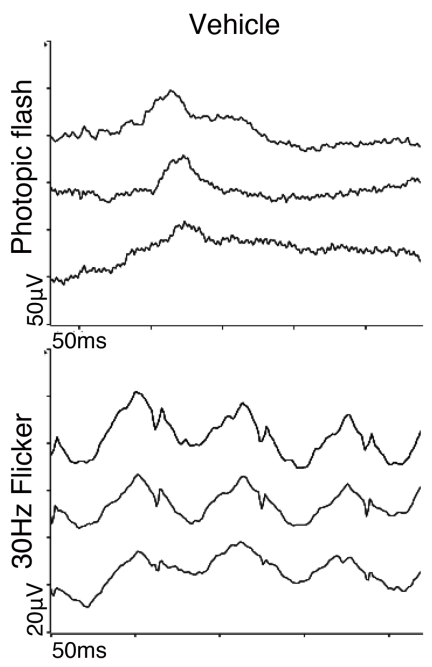

P45 [P17-P24]
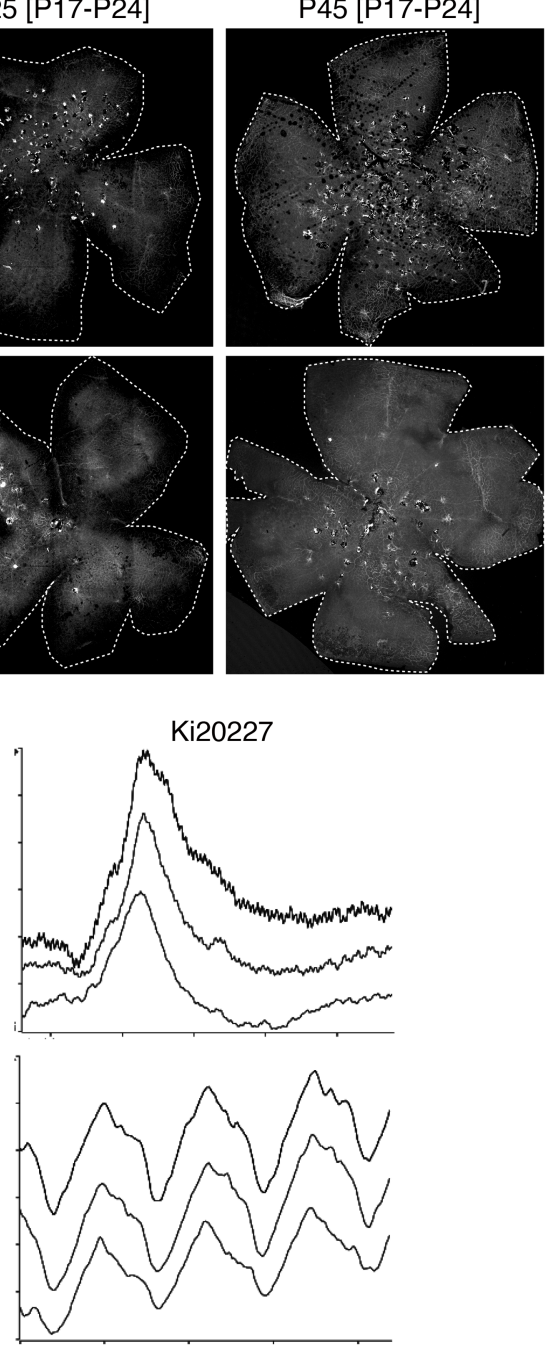

C

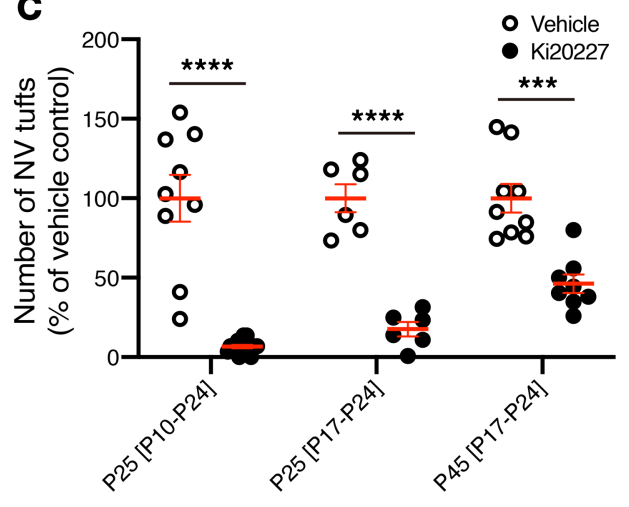

$\mathbf{E}$

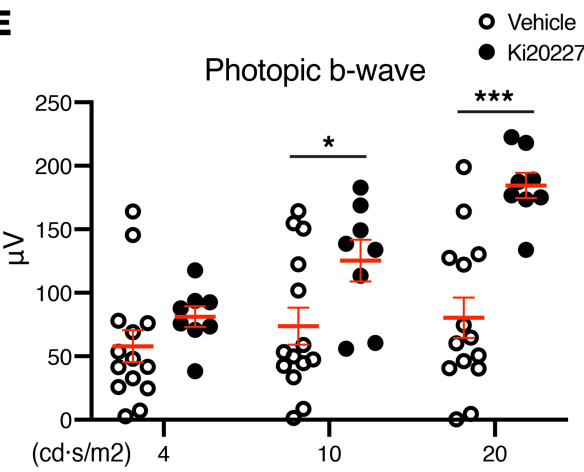

Flicker b-wave

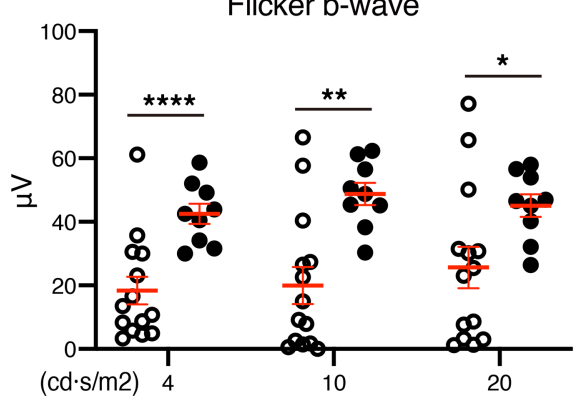

Figure 4. Microglia/macrophages ablation is sufficient to reduce NV and restore visual function in VIdIr/- mice. (A-C) The number of subretinal NV tufts in $\mathrm{VIdII}^{-1-}$; $\mathrm{CX} 3 \mathrm{Cr}^{\mathrm{CFP} /+}$ mice was analyzed at various time points after vehicle or Ki20227 treatment. (A) Three different time courses of treatment and the time points for analysis. (B) Subretinal NV tufts in retinal flat mount after Ki20227 treatment were visualized by GS-lectin. (C) The quantification of the number of subretinal NV tufts in 3 different time courses (P25 [P10-P24] vehicle: $n=9$, Ki20227: $n=15 ;$ P25 [P17-P24] vehicle: $n=6$, Ki20227: $n=6$; P45 [P17P24] vehicle: $n=9$, Ki20227: $n=8$ ). The $P$ values were calculated using multiple $t$ test. (D and E) Full-field ERGs were performed on P45 VIdIr ${ }^{-1-}$; $C \times 3 c r 7^{C F P /+}$ mice after treatment with vehicle or Ki20227 from P17 to 24. (D) The representative record of photopic flash ERG and photopic 30-Hz flicker ERG. (E) The amplitude of photopic flash and flicker b-wave were measured (vehicle: $n=14$, Ki20227: $n=8$ ). The $P$ values were calculated using multiple $t$ test. ${ }^{*} P<0.05$, ${ }^{* *} P<0.01,{ }^{* *} P<0.001,{ }^{* * * *} P<0.0001$. Error bars indicate the mean \pm SEM. Data represent at least 3 independent experiments. Scale bars: $1 \mathrm{~mm}$.

\section{Discussion}

We have demonstrated that ectopically distributed microglia were observed in the normally avascular photoreceptor layer, before the formation of subretinal NV tufts. Moreover, microglia ablation at early stages, before NV tufts emerged, by either chemical or genetic methods significantly inhibited the subretinal NV tuft formation in $\mathrm{Vldl}^{\prime-}$ mice. We have also demonstrated that treatment at later time points, after establishment of subretinal NV tufts, was sufficient to reduce NV and restore visual function. On the contrary, LPS-induced microglia activation increased the number of subretinal NV tufts. Taken together, these data demonstrate a critical role for retinal microglia/macrophages in the pathogenic phenotype of $\mathrm{Vldl}^{\prime-}$ mice. We have also demonstrated that the maculae of patients with MacTel Type2 exhibited a similar association 

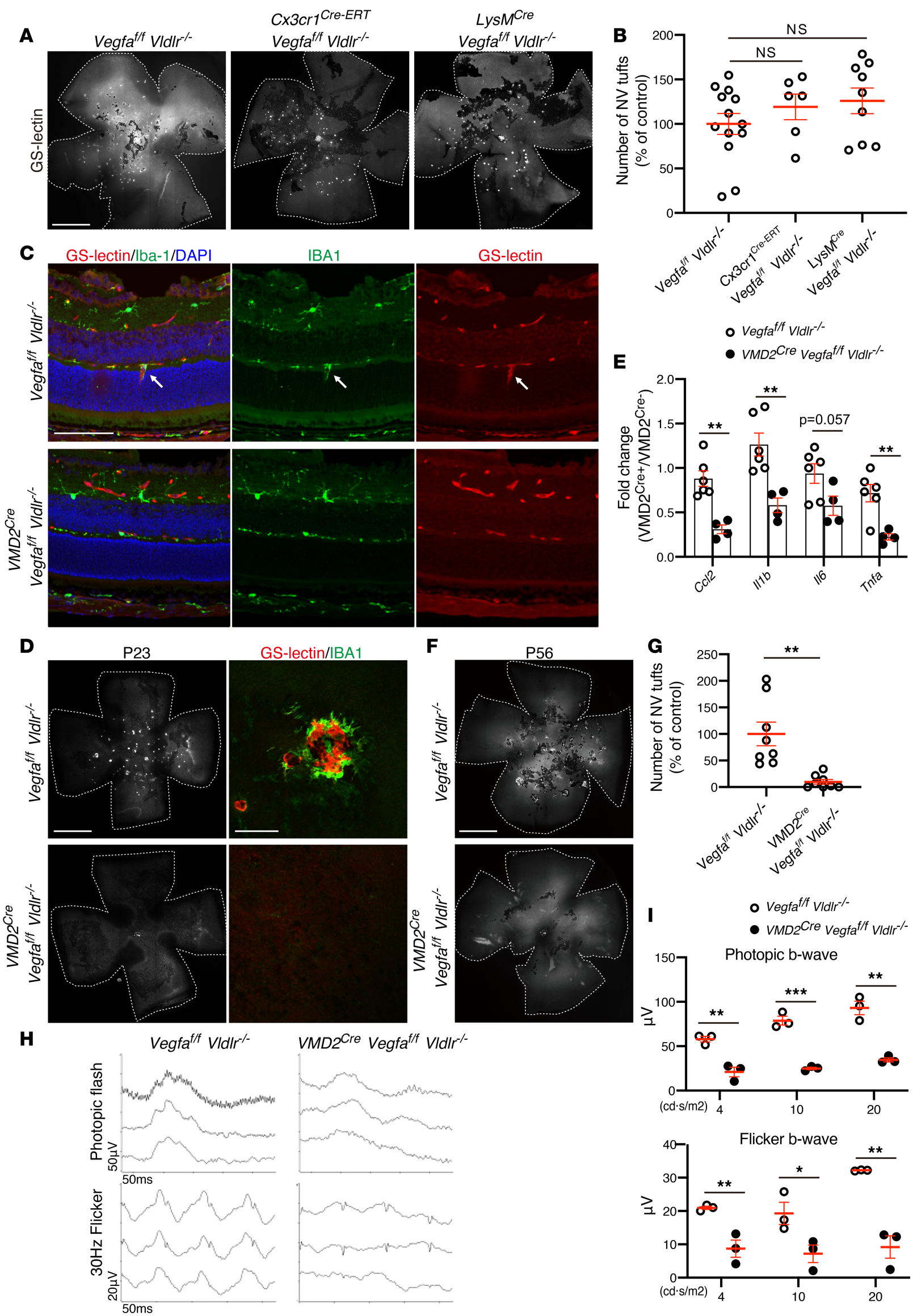
Figure 5. Vegfa deletion in RPE, but not microglia/macrophages, inhibits neovascular tufts and microglia activations but further reduces cone photoreceptor function in VIdIr/- mice. (A and B) To delete Vegfa expression in microglia of $V_{I d l r^{-1}}$ mice, VIdlr $r^{-1-}$ mice were crossed with microglia-specific Veg-

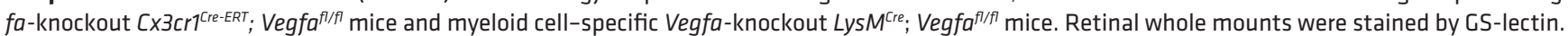

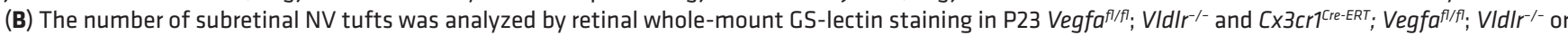

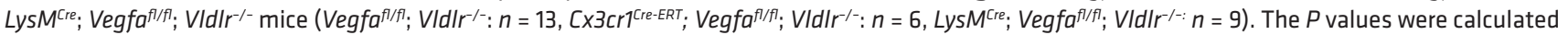
using 1-way ANOVA with Tukey's multiple comparisons test. (C-E) NV formation and microglia-related inflammatory cytokines and chemokines were analyzed in VIdl/r/- mice with RPE-specific Vegfa deletion. (C) Retinal sectional immunohistochemistry with GS-lectin and IBA-1 from P14 VMD2 $2^{\text {(re }}$; Vegfaf/fl; $\mathrm{VIdlr}^{-/-}$and Vegfa f/fli VIdIr $r^{-1-}$ mice. White arrows indicate coexistence of a retinal neovessel emerged from deep plexus and retinal microglia. (D) The retinal NV tufts at P23 were visualized by GS-lectin (left). The subretinal NV tufts were colocalized with IBA-1 microglia/macrophages (right). (E) The expression

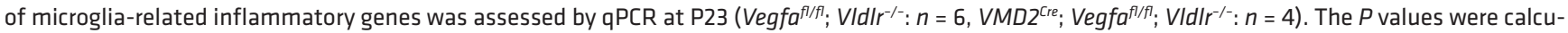
lated using multiple $t$ test. (F-I) The number of subretinal NV tufts and photopic ERGs was quantified in VIdlr-1- mice with RPE-specific gene deletion for

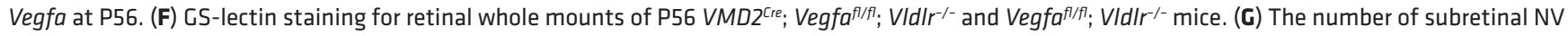
tufts was analyzed at P56 ( $n=8$ each). The $P$ values were calculated using an unpaired 2-tailed $t$ test. (H) The representative record of photopic flash ERG and photopic 30-Hz flicker ERG. (I) The amplitude of b-wave from photopic flash ERG and photopic 30-Hz flicker ERG were measured ( $n=3$ each). The $P$ values were calculated using multiple $t$ test. ${ }^{*} P<0.05$, ${ }^{*} P<0.01$, ${ }^{* * *} P<0.001$. Scale bars: $1 \mathrm{~mm}$ (A; D, left; and F); $100 \mu \mathrm{m}$ (C and $\mathbf{D}$, right).

between neovessels and microglia/macrophage as observed in Vldlr/- mice, suggesting that these findings are likely to correlate with human disease as well. It was also reported that retinal microglia accumulated in affected zones of the maculae of patients with AMD and located in close physical contact with choroidal NV (33). Collectively, these findings suggest that microglia associated with subretinal NV occurs in various human retinal diseases as well. We know earlier treatments with LPS (at P4) and Ki20227 (at P10) show maximum effects on the number of NV tufts compared with later treatment, and LPS treatment at P28 did not exacerbate the number of NV tufts. This implies that activated microglia are critical for sprouting of the $\mathrm{NV}$ tufts from deep retinal vasculature, which occurs in early stages of $\mathrm{Vldl}^{\prime-}$ mice.

We found that $\mathrm{Ccl} 2$ expression was highly upregulated in the $V l d l r^{-}$mouse retina at P12 before subretinal NV formation. $\mathrm{Ccl} 2$ is primarily secreted by activated microglia to recruit inflammatory monocytes expressing $C c r 2$, and the expression level of $C c l 2$ in retinas is strongly enhanced in retinal NV diseases such as AMD or proliferative diabetic retinopathy $(20,21,34)$. Furthermore, $C c l 2$ has also been shown to directly induce $\mathrm{NV}(35,36)$ in retinas. The induction of $\mathrm{Ccl} 2 \mathrm{in} \mathrm{Vldl}^{-1}$ mice again implicates microglia in the associated pathological phenotype. This was further corroborated by the fact that inhibition of CSF-1R, also key to microglia/macrophage recruitment, resulted in a dramatic reduction in $\mathrm{NV}$ tuft formation.

The simplest explanation for why microglia/macrophage ablation improves retinal function is prevention of microglia depletion. Additionally, inactivation by CSF-1R inhibition could prevent photoreceptor degeneration, which may normally occur through the release of neurotoxic compounds during phagocytosis of cell debris and aggregated proteins.

It is known that microglia and macrophages have important roles in both physiological and pathological angiogenesis in various CNS diseases, such as brain tumor, ischemic stroke, and neurodegenerative diseases. Microglia/macrophages promote NV via secretion of Ccl2, Tnfa, Illb, Vegfa, or MMPs $(35,41,42)$. We have demonstrated that Vegfa deletion in microglia/macrophages did not rescue the $\mathrm{NV}$ phenotype of $V l d l r^{-1}$ mice, suggesting that microglia promote $\mathrm{NV}$ tuft formation by secreting other non-Vegfa factors, such as Ccl2, Tnfa, Illb, or MMPs, which can also induce NV. However, as expected, $V e g f a$ is still a key driver of the subretinal NV phenotype. We detected that Vegfa is strongly enriched in the outer retinas of $\mathrm{Vldlr}^{-}$mice, as observed by ISH, compared with expression that is mainly limited to the INL during development of normal retinas (43). This correlates well with previous studies that demonstrated high levels of VEGF expression in the ONL and RPE of $\operatorname{Vldlr^{-1}}$ mice $(15,19,32)$. Indeed, Vegfa deletion in the RPE of Vldlr ${ }^{--}$mice significantly blocked subretinal $\mathrm{NV}$ formations by eliminating microglia migration into the subretinal space and reducing microglia-related inflammatory and angiogenic genes, such as $C c l 2$, Tnfa, or Il1b. Previously, Joyal et al. have reported impaired uptake of both glucose and lipids into photoreceptors of $\mathrm{Vldlr}^{-1-}$ mice, leading to a shortage of the Krebs cycle intermediate $\alpha$-ketoglutarate, which subsequently promotes stabilization of hypoxia-induced factor $1 \mathrm{a}$ and secretion of Vegfa (44). Sun et al. showed that VEGF upregulation by c-fos in the Vldlr ${ }^{\prime-}$ photoreceptor controls retinal NV growth into the normally avascular photoreceptor layer (19). We have shown that Vegfa deletion from RPE significantly suppressed microglia/macrophage migration and activation

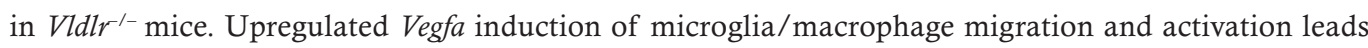
to subsequent abnormal NV. In fact, it has been reported that microglia/macrophages express VegfrI 
and Vegfa serves as a chemoattractant for microglia, inducing their subsequent migration, activation, and participate in the angiogenic response $(45,46)$. Collectively, we suggest that Vegfa upregulation in photoreceptors or RPE induce microglial activation or migration, after which $C c l 2$ is secreted by activated microglia leading to the recruitment of more microglia/macrophages. This process ultimately promotes retinal neovessel growth into the normally avascular outer retina in Vldlr ${ }^{--}$mice. Although Vegfa deletion in RPE in $\mathrm{Vldl}^{-1}$ mice inhibited retinal NV tufts significantly, cone photoreceptor function in these mice was even worse than in $V l d l r^{-1}$ mice, consistent with previous reports that endogenous Vegfa provides critical trophic support necessary for retinal function (10). Thus, we suggest that targeting only Vegfa is neither an effective nor a safe strategy for treating vascular diseases of the macula, such as $\mathrm{MacTel}$, and that regulating microglia/macrophage may represent a more effective and safer strategy for preventing subretinal NV.

As $C \times 3$ cr 1 does not distinguish retinal resident microglia from circulating monocyte-derived macrophages, we can not further delineate the roles of macrophages or microglia in this study.

Understanding the role of microglia in $\mathrm{Vldl}^{-1}$ mice may help provide clues for how subretinal NV occurs in patients. Microglia closely associate with NV tufts in patients with MacTel in a manner reminiscent of $\mathrm{Vldl}^{-1-}$ mice. Thus, learning how to therapeutically modulate microglia activity in the CNS may provide broad benefits in treating multiple CNS disorders.

\section{Methods}

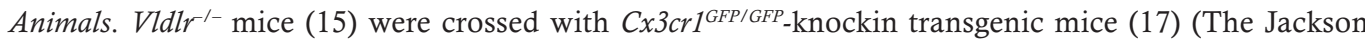
Laboratory) expressing GFP under the control of the chemokine (C-X3-C motif) receptor 1 ( $C x 3 \mathrm{cr} 1)$ promoter, which drives expression in microglia/macrophages (17) to generate $V l d l r^{-1}, C x 3 \mathrm{cr} 1^{G F P /+}$ mice, with GFP expression in retinal macrophages/microglia within the Vldlr ${ }^{-1}$ background. CX3cr1 $1^{\text {CreERT }}$ (47) mice (The Jackson Laboratory) expressing tamoxifen-inducible Cre recombinase or M-lysozyme

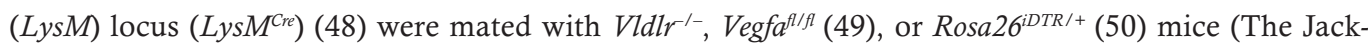
son Laboratory) to generate conditional gene knockout mice and permit macrophage and microglia

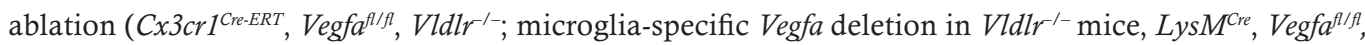

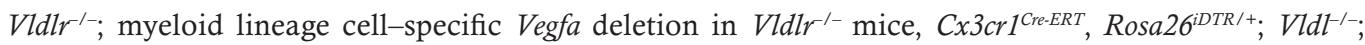
genetical microglia depletion in Vldlr ${ }^{-1}$ mice). To induce $C x 3 c r 1$-Cre recombination, $0.1 \mathrm{mg} / \mathrm{g}$ body weight of 4-OHT (MilliporeSigma)/cone oil solution was administered to Cx3cr1 ${ }^{\text {CreERT; }}$ Vegfa $^{f l f l}$; Vldll ${ }^{-1}$, and control littermates ( $V e g f a^{f l f l} ; V_{l d l r^{-1}}$ ) subcutaneously once a day from P12 to P14. For CX3cr $1^{\text {Cre-ERT; }}$ Rosa26 $6^{i D T R /+} ; V_{l d l r^{-1}}$ mice, $0.1 \mathrm{mg} / \mathrm{g}$ body weight of 4-OHT was injected subcutaneously at P10 and P11 and $20 \mathrm{ng} / \mathrm{g}$ body weight of DT (MilliporeSigma) was injected intraperitoneally from P12 to P14. They were analyzed at P23. Transgenic mice carrying the human vitelliform macular dystrophy-2 (VMD2) promoter-directed reverse tetracycline-dependent transactivator and the tetracycline-responsive element-directed (TRE-directed) Cre recombinase $\left(V M D 2^{\text {re }}\right)(51)$ were mated with $V l d l r^{-1}$, Vegfallft mice (49) (VMD2 $2^{\text {Cre }} V e g f a^{f l f l}$; Vldl ${ }^{\prime-}$; retinal RPE-specific Vegfa deletion in Vldl ${ }^{\prime-}$ mice). To induce gene deletion, $80 \mathrm{mg} / \mathrm{g}$ body weight of doxycycline (MilliporeSigma) was injected daily intraperitoneally for consecutive 3 days and then analyzed by immunohistochemistry or ERG at varying time points, as indicated in the Results and the legend for Figure 5. Littermates harboring floxed alleles but no Cre-recombinase were used as control. To deplete microglia/macrophages, the CSF-1R inhibitor (Ki20227)

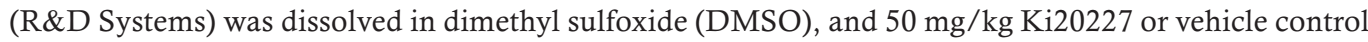
(DMSO) was injected intraperitoneally on a daily basis as previously described (24). To activate microglia/macrophages, $1 \mathrm{mg} / \mathrm{kg}$ of LPS (MilliporeSigma) or control vehicle (PBS) was injected intraperitoneally into $\mathrm{Vldl}^{-1}$ mice at P4 or P28.

Human samples. The donor eye from a patient with MacTel type 2 (52-year-old female) was obtained as previously described (52). The time between death and fixation of the MacTel eye in $4 \%$ paraformaldehyde was just over 4 hours. The fixed retina was dehydrated and sectioned from paraffin wax, and immunohistochemistry was performed.

Immunofluorescence. Retinas or RPE/choroid complexes were dissected and prepared for whole mounts or sectioning. For preparation of retinal cross-sections, isolated eyes were fixed in 4\% PFA for 4 hours, placed in $20 \%$ sucrose for 4 hours at $4^{\circ} \mathrm{C}$, and embedded in Tissue-Tek OCT compound (Sakura Finetek) for subsequent cryosectioning. For preparation of whole mounts, after fixation in 4\% PFA for 1 hour, retinas or RPE/choroid complexes were dissected and laid flat with 4 radial relaxing incisions. Whole-mount 
retinas, $\mathrm{RPE} /$ choroid complexes, or cryosections were incubated in blocking buffer (PBS with 10\% fetal bovine serum, $10 \%$ normal goat serum, and $0.1 \%$ Triton $\mathrm{X}-100$ ) for 2 hours at $4^{\circ} \mathrm{C}$, following by an overnight incubation with primary antibodies in blocking buffer at $4^{\circ} \mathrm{C}$. Tissue specimens were then washed and incubated with the corresponding Alexa Fluor-conjugated secondary antibodies (Thermo Fisher) for 3 hours, and nuclei were stained with DAPI (Vector Laboratories). After staining, they were washed and mounted in ProLong Diamond Antifade mounting medium (Thermo Fisher). Retinal whole mounts were mounted with the photoreceptor side down. Primary antibodies targeting ZO-1 (33-9100/ZO1-1A12, Thermo Fisher), CD68 (MCA-1957/FA-11, Bio-Rad), Collagen Type 4 (AB769, Millipore Sigma), and Iba-1 (019-19741, Wako) were used. Fluorescent-conjugated isolectin Griffonia Simplicifolia IB-4 (GS-Lectin) (I21412, Thermo Fisher) was also used for labeling endothelial cells. All images were acquired with a confocal laser scanning microscope (LSM 710, Zeiss) and processed with the ZEN 2010 software (Zeiss). Three-dimensional reconstructions were generated using ZEN 2010 and Imaris software (Bitplane). The quantification of GFP-positive pixels and the number of NV tufts was performed by NIH ImageJ software.

Gene expression analyses. For quantitative PCR, single retinas were collected in $500 \mu 1$ Trizol, and RNA was isolated using a Purelink RNA mini kit (Thermo Fisher) according to the manufacturer's instructions. 700 ng RNA was used for RT-qPCR using the high-capacity cDNA reverse transcription kit (Thermo Fisher). qPCR was performed using TaqMan universal master mix (Thermo Fisher) and TaqMan probes on a Quantstudio 5 (Thermo Fisher). $\beta$-Actin was used as the reference gene. For PCR array, total RNA was prepared from P12 $\mathrm{Vldl}^{-/}$and $\mathrm{Vldlr}^{\prime+}$ retinas using the RNeasy Plus Mini kit (QIAGEN) and was reverse transcribed using the $\mathrm{RT}^{2}$ First Strand cDNA Kit (QIAGEN). mRNA PCR array for angiogenesis (RT ${ }^{2}$ Profiler PCR Array for Mouse Angiogenesis [PAMM-024]) was used according to the manufacturer's instructions (QIAGEN). Data were analyzed with 7900 HT Fast System SDS 2.4 Software (Thermo Fisher).

In vivo imaging. Confocal scanning laser ophthalmoscopy detecting fundus autofluorescence by infrared reflectance and ICG were performed using a Spectralis imaging platform (Heidelberg Engineering). Mice were anesthetized by intraperitoneal injection of $100 \mathrm{mg} / \mathrm{kg}$ ketamine and $10 \mathrm{mg} / \mathrm{kg}$ xylazine and perfused with indocyanine green $(50 \mu \mathrm{g} / \mathrm{g}$ body weight). Pupils were dilated with $1 \%$ tropicamide and $2.5 \%$ phenylephrine before imaging.

Ganzfeld ERGs. The phenotypes of the mice were characterized using Ganzfeld ERGs and immunohistochemistry as previously described $(43,52)$. Mice were dark-adapted overnight before the experiments and anesthetized under a dim red light by intraperitoneal injection of $100 \mathrm{mg} / \mathrm{kg}$ ketamine and $10 \mathrm{mg} / \mathrm{kg}$ xylazine. Silver needle electrodes served as reference (forehead) and ground (tail). Full-field ERGs were recorded from the corneal surface of each eye after pupil dilation (with $1 \%$ tropicamide and $2.5 \%$ phenylephrine) with active contact lens electrodes (Mayo) using a computerized system with an electronically controlled Ganzfeld dome (Espion E2 with Colordome; Diagnosys). For photopic (light-adapted) measurements, a $30 \mathrm{~cd} / \mathrm{m}^{2}$ background was used, and cone responses to $1-\mathrm{Hz}\left(0.63-20 \mathrm{~cd} \cdot \mathrm{s} / \mathrm{m}^{2}\right)$ and $30-\mathrm{Hz}\left(3.98,10\right.$, and $\left.20 \mathrm{~cd} \cdot \mathrm{s} / \mathrm{m}^{2}\right)$ flicker stimuli were recorded. All ERG responses were filtered at $0.3-500 \mathrm{~Hz}$, and signal averaging was applied.

ISH. ISH was performed using ViewRNA ISH Tissue Assay for RNA kits (catalog QVT0013, Affymetrix) according to the manufacturer's instructions. The ISH probe for mouse Vegfa and Itgam was designed and synthesized by Affymetrix (Vegfa, VB1-12654; Itgam, VB4-10683; Affymetrix).

Statistics. All statistical tests were performed in GraphPad Prism 8. Data comparisons between 2 groups were performed using unpaired 2-tailed Student's $t$ tests or multiple 2-tailed $t$ tests. Data comparisons between multiple groups were performed with 1-way ANOVA with Tukey's correction. Statistical tests used for each experiment are specified in the figure legends. Data are represented as mean \pm SEM. $P<0.05$ was considered statistically significant.

Study approval. All animal experimental procedures were approved by The Scripps Research Institute Animal Care and Use Committee. All experiments were performed in accordance with the NIH Guide for the Care and Use of Laboratory Animals (National Academies Press, 2011). For the human sample, the donor eye from a patient with MacTel type 2 (52-year-old female) was obtained as previously described (52). Informed consent and approval from The Scripps Research Institute IRB were obtained in accordance with the Declaration of Helsinki.

\section{Author contributions}

YU, TK, and AUO designed the study and planned the experiments. AUO, YU, TK, EA, YI, SS, and SB performed the experiments. AUO, YU, and TK analyzed the data. AUO, YU, MID, and MF wrote the manuscript. 


\section{Acknowledgments}

We thank Marcus Fruttiger for providing the retinal images from the patient with MacTel. We thank Mauricio Rosenfeld, Stacey K. Moreno, and Maki Kitano for excellent technical assistance and discussion. This work was supported by grants to MF from the National Eye Institute (EY-022025-01) and the Lowy Medical Research Institute (MacTel). AUO is supported by a fellowship from the Manpei Suzuki Diabetes Foundation and JSPS KAKENHI grant 17K16984. YU was supported by JSPS KAKENHI grant 16K11330 and research grants from Bayer Yakuhin Ltd. and Public Interest Foundation for the Elderly Eye Diseases Research Foundation.

Address correspondence to: Martin Friedlander, The Scripps Research Institute, MB 216, 10550 North Torrey Pines Road, La Jolla, California 92037, USA. Phone: 858.784.9138; Email: friedlan@scripps.edu.

1. Usui Y, et al. Angiogenesis and Eye Disease. Annu Rev Vis Sci. 2015;1:155-184.

2. Miller JW. Age-related macular degeneration revisited--piecing the puzzle: the LXIX Edward Jackson memorial lecture. Am J Ophthalmol. 2013;155(1):1-35.e13.

3. Gass JD, Blodi BA. Idiopathic juxtafoveolar retinal telangiectasis. Update of classification and follow-up study. Ophthalmology. 1993;100(10):1536-1546.

4. Yannuzzi LA, et al. Retinal angiomatous proliferation in age-related macular degeneration. Retina (Philadelphia, Pa). 2001;21(5):416-434.

5. Yannuzzi LA, Bardal AM, Freund KB, Chen KJ, Eandi CM, Blodi B. Idiopathic macular telangiectasia. Arch Ophthalmol. 2006;124(4):450-460

6. Usui-Ouchi A, Friedlander M. Anti-VEGF therapy: higher potency and long-lasting antagonism are not necessarily better. $J$ Clin Invest. 2019;129(8):3032-3034

7. Keir L, Moorsel Fv, Saleem MA, Richards A. Beware renal adverse effects of anti-vascular endothelial growth factor treatment. BMJ. 2012;344:e3838.

8. Grunwald JE, et al. Risk of geographic atrophy in the comparison of age-related macular degeneration treatments trials. Ophthalmology. 2014;121(1):150-161.

9. Kurihara T, et al. Hypoxia-induced metabolic stress in retinal pigment epithelial cells is sufficient to induce photoreceptor degeneration. Elife. 2016;5:e14319.

10. Kurihara T, Westenskow PD, Bravo S, Aguilar E, Friedlander M. Targeted deletion of Vegfa in adult mice induces vision loss. J Clin Invest. 2012;122(11):4213-4217.

11. Sigler EJ, Randolph JC, Calzada JI, Charles S. Comparison of observation, intravitreal bevacizumab, or pars plana vitrectomy for non-proliferative type 2 idiopathic macular telangiectasia. Graefes Arch Clin Exp Ophthalmol. 2013;251(4):1097-1101.

12. Charbel Issa P, Finger RP, Kruse K, Baumüller S, Scholl HP, Holz FG. Monthly ranibizumab for nonproliferative macular telangiectasia type 2: a 12-month prospective study. Am J Ophthalmol. 2011;151(5):876-886.e1.

13. Gharbiya M, Parisi F, Cruciani F, Bozzoni-Pantaleoni F, Pranno F, Abdolrahimzadeh S. Intravitreal anti-vascular endothelial growth factor for retinal angiomatous proliferation in treatment-naive eyes: long-term functional and anatomical results using a modified PrONTO-style regimen. Retina (Philadelphia, Pa). 2014;34(2):298-305.

14. Heckenlively JR, et al. Mouse model of subretinal neovascularization with choroidal anastomosis. Retina (Philadelphia, Pa). 2003;23(4):518-522.

15. Dorrell MI, et al. Antioxidant or neurotrophic factor treatment preserves function in a mouse model of neovascularization-associated oxidative stress. J Clin Invest. 2009;119(3):611-623.

16. Ritter MR, Banin E, Moreno SK, Aguilar E, Dorrell MI, Friedlander M. Myeloid progenitors differentiate into microglia and promote vascular repair in a model of ischemic retinopathy. J Clin Invest. 2006;116(12):3266-3276.

17. Jung S, et al. Analysis of fractalkine receptor CX(3)CR1 function by targeted deletion and green fluorescent protein reporter gene insertion. Mol Cell Biol. 2000;20(11):4106-4114.

18. Rathnasamy G, Foulds WS, Ling EA, Kaur C. Retinal microglia - A key player in healthy and diseased retina. Prog Neurobiol. 2019;173:18-40.

19. Sun Y, et al. Inflammatory signals from photoreceptor modulate pathological retinal angiogenesis via c-Fos. J Exp Med. 2017;214(6):1753-1767.

20. Akhtar-Schäfer I, Wang L, Krohne TU, Xu H, Langmann T. Modulation of three key innate immune pathways for the most common retinal degenerative diseases. EMBO Mol Med. 2018;10(10):e8259.

21. Sennlaub F, et al. CCR2(+) monocytes infiltrate atrophic lesions in age-related macular disease and mediate photoreceptor degeneration in experimental subretinal inflammation in Cx3cr1 deficient mice. EMBO Mol Med. 2013;5(11):1775-1793

22. Elmore MR, et al. Colony-stimulating factor 1 receptor signaling is necessary for microglia viability, unmasking a microglia progenitor cell in the adult brain. Neuron. 2014;82(2):380-397.

23. Okunuki Y, et al. Retinal microglia initiate neuroinflammation in ocular autoimmunity. Proc Natl Acad Sci USA. 2019;116(20):9989-9998.

24. Kubota Y, et al. M-CSF inhibition selectively targets pathological angiogenesis and lymphangiogenesis. J Exp Med. 2009;206(5):1089-1102.

25. Liddelow SA, et al. Neurotoxic reactive astrocytes are induced by activated microglia. Nature. 2017;541(7638):481-487.

26. Bennett ML, et al. New tools for studying microglia in the mouse and human CNS. Proc Natl Acad Sci USA. 2016;113(12):E1738-E1746. 
27. Chen Y, Hu Y, Lu K, Flannery JG, Ma JX. Very low density lipoprotein receptor, a negative regulator of the wnt signaling pathway and choroidal neovascularization. J Biol Chem. 2007;282(47):34420-34428.

28. Shi J, Hua L, Harmer D, Li P, Ren G. Cre Driver Mice Targeting Macrophages. Methods Mol Biol. 2018;1784:263-275.

29. Dejda A, et al. Neuropilin-1-Expressing Microglia Are Associated With Nascent Retinal Vasculature Yet Dispensable for Developmental Angiogenesis. Invest Ophthalmol Vis Sci. 2016;57(4):1530-1536.

30. Goldmann T, et al. Origin, fate and dynamics of macrophages at central nervous system interfaces. Nat Immunol. 2016;17(7):797-805.

31. Mendes-Jorge L, et al. Scavenger function of resident autofluorescent perivascular macrophages and their contribution to the maintenance of the blood-retinal barrier. Invest Ophthalmol Vis Sci. 2009;50(12):5997-6005.

32. Hua J, et al. Resveratrol inhibits pathologic retinal neovascularization in Vldlr(-/-) mice. Invest Ophthalmol Vis Sci. 2011;52(5):2809-2816

33. Combadière C, et al. CX3CR1-dependent subretinal microglia cell accumulation is associated with cardinal features of age-related macular degeneration. J Clin Invest. 2007;117(10):2920-2928.

34. Taghavi Y, Hassanshahi G, Kounis NG, Koniari I, Khorramdelazad H. Monocyte chemoattractant protein-1 (MCP-1/CCL2) in diabetic retinopathy: latest evidence and clinical considerations. J Cell Commun Signal. 2019;13(4):451-462.

35. Stamatovic SM, Keep RF, Mostarica-Stojkovic M, Andjelkovic AV. CCL2 regulates angiogenesis via activation of Ets-1 transcription factor. J Immunol. 2006;177(4):2651-2661.

36. Ridiandries A, Tan JT, Bursill CA. The Role of CC-Chemokines in the Regulation of Angiogenesis. Int J Mol Sci. 2016;17(11):E1856

37. Harada T, et al. Microglia-Müller glia cell interactions control neurotrophic factor production during light-induced retinal degeneration. J Neurosci. 2002;22(21):9228-9236.

38. Srinivasan B, Roque CH, Hempstead BL, Al-Ubaidi MR, Roque RS. Microglia-derived pronerve growth factor promotes photoreceptor cell death via p75 neurotrophin receptor. J Biol Chem. 2004;279(40):41839-41845.

39. Kaur C, Rathnasamy G, Ling EA. Roles of activated microglia in hypoxia induced neuroinflammation in the developing brain and the retina. J Neuroimmune Pharmacol. 2013;8(1):66-78.

40. Schwartz M, Shechter R. Systemic inflammatory cells fight off neurodegenerative disease. Nat Rev Neurol. 2010;6(7):405-410.

41. Zhao X, Eyo UB, Murugan M, Wu LJ. Microglial interactions with the neurovascular system in physiology and pathology. Dev Neurobiol. 2018;78(6):604-617.

42. Brandenburg S, et al. Resident microglia rather than peripheral macrophages promote vascularization in brain tumors and are source of alternative pro-angiogenic factors. Acta Neuropathol. 2016;131(3):365-378.

43. Usui Y, et al. Neurovascular crosstalk between interneurons and capillaries is required for vision. J Clin Invest. 2015;125(6):2335-2346.

44. Joyal JS, et al. Retinal lipid and glucose metabolism dictates angiogenesis through the lipid sensor Ffar1. Nat Med. 2016;22(4):439-445

45. Forstreuter F, Lucius R, Mentlein R. Vascular endothelial growth factor induces chemotaxis and proliferation of microglial cells. J Neuroimmunol. 2002;132(1-2):93-98.

46. Kerber M, et al. Flt-1 signaling in macrophages promotes glioma growth in vivo. Cancer Res. 2008;68(18):7342-7351.

47. Parkhurst CN, et al. Microglia promote learning-dependent synapse formation through brain-derived neurotrophic factor. Cell. 2013;155(7):1596-1609.

48. Clausen BE, Burkhardt C, Reith W, Renkawitz R, Förster I. Conditional gene targeting in macrophages and granulocytes using LysMcre mice. Transgenic Res. 1999;8(4):265-277.

49. Gerber HP, et al. VEGF is required for growth and survival in neonatal mice. Development. 1999;126(6):1149-1159.

50. Buch T, et al. A Cre-inducible diphtheria toxin receptor mediates cell lineage ablation after toxin administration. Nat Methods. 2005;2(6):419-426.

51. Le YZ, et al. Inducible expression of cre recombinase in the retinal pigmented epithelium. Invest Ophthalmol Vis Sci. 2008;49(3):1248-1253

52. Powner MB, Gillies MC, Zhu M, Vevis K, Hunyor AP, Fruttiger M. Loss of Müller's cells and photoreceptors in macular telangiectasia type 2. Ophthalmology. 2013;120(11):2344-2352. 